\title{
Channel Sounding for Multi-User Massive MIMO in Distributed Antenna System Environment
}

\author{
Seoyoung $\mathrm{Yu}^{+}+\mathbb{1}$ and Jeong Woo Lee $*++(1)$ \\ School of Electrical and Electronics Engineering, Chung-Ang University, Seoul 06974, Korea; \\ holywillow@naver.com \\ * Correspondence: jwlee2@cau.ac.kr; Tel.: +82-2-820-5734 \\ +Current address: 84 Heukseok-ro, Dongjak-gu, Seoul 06974, Korea.
}

Received: 20 November 2018; Accepted: 21 December 2018; Published: 1 January 2019

\begin{abstract}
We propose a generation scheme for a sounding reference signal (SRS) suitable for supporting a large number of users in massive multi-input multi-output (MIMO) system with a distributed antenna system (DAS) environment. The proposed SRS can alleviate the pilot contamination problem which occurs inherently in the multi-user system due to the limited number of orthogonal sequences. The proposed SRS sequence is generated by applying a well-chosen phase rotation to the conventional LTE/LTE-A SRS sequences without requiring an increased amount of resource usage. We also propose using the correlation-aided channel estimation algorithm as a supplemental scheme to obtain more reliable and refined channel estimation. It is shown that the proposed SRS sequence and the supplemental channel estimation scheme improve significantly the channel estimation performance in multi-user massive MIMO systems.
\end{abstract}

Keywords: massive multi-input multi-output (MIMO); distributed antenna systems (DAS); sounding reference signal (SRS); channel estimation

\section{Introduction}

It is expected that the amount of mobile wireless traffic in 2020 will be 1000 times higher than that of 2010 [1-3]. Along with the dramatic growth in the demand for wireless communications, performance requirements for data rate, spectral efficiency and energy efficiency are also getting higher [4-6]. To meet these growing demand and performance requirements for wireless communications, massive multi-input multi-output (m-MIMO) technology was proposed as one of the key technologies for the next generation cellular networks, known as fifth generation (5G) systems [7-10]. It is known that m-MIMO systems, whose transmitter or receiver is equipped with massive number of antennas, can improve the spectral efficiency and save energy in wireless communication systems [10-12]. As a result, $\mathrm{m}$-MIMO systems have recently attracted many researchers and engineers in many aspects. Multi-user m-MIMO technology, in which a base station (BS) uses a large number of antennas to serve many pieces of user equipment (UE) simultaneously on the same time-frequency resource, is one example that is actively studied to be practically adopted in 5G systems [13-15]. The distributed antenna system (DAS) has also been considered a key technology for feasible deployment of 5G systems [16-18]. In DAS configuration, there exist a digital unit (DU) and multiple radio units (RUs) in a cell, where each RU is connected to DU via fiber optic links. The DU manages RUs in a centralized manner, by which RUs can transmit and receive signals in a cooperative manner.

The key requirement for enjoying the benefit of m-MIMO technology is to obtain the accurate channel state information (CSI) for each link at the BS, or at RUs in DAS environment. In the frequency-division duplex (FDD) approach, UEs estimate downlink (DL) channels by using DL pilot signals, or sounding reference signals (SRS), transmitted from the BS [19]. The required number 
of DL pilots in an FDD based approach is proportional to the number of BS antennas multiplied by the number of served UEs, which complicates the adoption of such a DL channel estimation in massive MIMO environments. Thus, the m-MIMO systems typically employ the time-division duplex (TDD) approach to estimate the DL channel. In the TDD approach, UEs estimate DL channels by sending mutually orthogonal uplink (UL) pilot signals (SRS) to BS based on the DL and UL channel reciprocity within the channel coherence interval [9]. The total number of UL pilots required in such a TDD based approach is proportional to the number of served UEs irrespective of the number of BS antennas [7,9].

For a given length of sequence, say $M$, we may generate at most $M$ orthogonal sequences. Conventionally, pilot sequences are mutually orthogonal, so the maximum number of pilot sequences is limited by $M$. If the number of UEs in simultaneous service exceeds $M$, we need to reuse some or all of the already generated orthogonal sequences. This results in the so-called pilot contamination problem [20] due to the violation of orthogonality between pilot sequences. The pilot contamination mainly limits the performance improvement of m-MIMO systems [7,15]. Most of the prior works that proposed to resolve this problem have considered the use of mutually orthogonal SRS sequences. They include pilot signal coordination [21], blind channel estimation with data samples [22] and cooperative multi-cell precoding in m-MIMO systems [23]. The number of mutually orthogonal SRS sequences is mainly limited by the length of the base sequence. In Long Term Evolution (LTE)/Long Term Evolution-Advanced (LTE-A) systems, the number of orthogonal SRS sequences is 16, which is not suitable for serving a large number of users in m-MIMO environments [24,25]. Using long pilot sequences may reduce the pilot contamination, but they replace data sequences and reduce channel spectral efficiency and throughput. If pilot sequences are made too long, they may occupy even the restricted band, which must be prevented. Thus, it may be desirable to generate a larger number of SRS sequences without increasing the sequence length.

For this purpose, we propose a mechanism for generating SRS sequences with a lower level of pilot contamination which is suitable for serving a large number of UEs. In the proposed mechanism, a phase rotation is applied to the base sequence without increasing the sequence length. The resultant SRS sequences may be mutually correlated and thus still incur a pilot contamination as the number of UEs grows and exceeds the length of base sequence. This results in high channel estimation error when a linear estimation based on the orthogonality of SRS sequences is used. To resolve this problem, we propose a two-step channel estimation algorithm by which the least square (LS) estimation [26] is first applied and the minimum mean squared error (MMSE) estimation [27] is additionally applied only to the group of UEs using mutually correlated SRS sequences. The proposed correlation-aided channel estimation shows the improved performance in the channel estimation. It is shown that the proposed SRS combined with the supplemental channel estimation algorithm guarantees lower mean squared error (MSE) in channel estimation, which alleviates the pilot contamination problem.

The rest of the paper is organized as follows. In Section 2, we introduce the system model in consideration. In Section 3, a brief review of SRS sequences in conventional LTE/LTE-A systems is provided. We introduce the generation of proposed SRS sequences and the analysis on the resultant correlation in Section 4. In Section 5, we propose the two-step channel estimation algorithm composed of an LS estimation followed by a supplemental MMSE estimation. We also formulate and analyze the MSE obtained for SRS sequences with an LS estimation. We verify the performances in various aspects by computer simulations in Section 6 and conclude this paper in Section 7.

\section{System Model}

Consider a cell having a DU and $R$ RUs each of which serves $K$ UEs as shown in Figure 1 , where each RU has $N_{T}$ transmit and receive antennas. We index RU by $r \in\{0, \cdots, R-1\}$, and index the UE served by RU $r$ as $r(k), k \in\{0, \cdots, K-1\}$. We consider orthogonal frequency division multiplexing (OFDM) communications with $N_{c}$ subcarriers between UE and RU. We suppose the channel reciprocity, by which the DL channel from RU to UE can be estimated by using the UL pilots sent from UEs under the constraint that the time delay from the UL channel estimation to the DL 
transmission is less than the coherence time of the channel [7]. Then, the UL channel estimated by sending SRS sequence from each UE to RU is used as the DL channel estimation. Let $\mathbf{s}_{r(k)}=$ $\left[s_{r(k)}[0] \cdots s_{r(k)}[M-1]\right]^{T}$ denote the SRS sequence of UE $r(k)$, where the superscript $T$ denotes a transpose of a vector. We also let $\mathbf{h}_{r(k), r^{\prime}}[m] \in \mathcal{C}^{N_{T} \times 1}$ denote the channel gain between UE $r(k)$ and RU $r^{\prime}$ over the $m$-th subcarrier. Then, the channel gains corresponding to $M$ subcarriers, $\mathbf{h}_{r(k), r^{\prime}}[m]$, $m=0, \cdots, M-1$, are estimated by using an SRS sequence.

The signal received by RU $r$ over the subcarrier $m$ is denoted by $\mathbf{y}_{r}[m] \in \mathcal{C}^{N_{T} \times 1}$ and obtained by

$$
\mathbf{y}_{r}[m]=\sum_{k=0}^{K-1} \mathbf{h}_{r(k), r}[m] s_{r(k)}[m]+\sum_{r^{\prime}=0, r^{\prime} \neq r}^{R-1} \sum_{k=0}^{K-1} \mathbf{h}_{r^{\prime}(k), r}[m] s_{r^{\prime}(k)}[m]+\mathbf{n}_{r}[m]
$$

where $\mathbf{n}_{r}[m] \in \mathcal{C}^{N_{T} \times 1}$ is the zero-mean additive white Gaussian noise vector with covariance matrix $\sigma_{n}^{2} \mathbf{I}_{N_{T} \times N_{T}}$. Note that $\mathbf{h}_{r(k), r^{\prime}}[m]=\beta_{r(k), r^{\prime}} \mathbf{g}_{r(k), r^{\prime}}[m]$, where $\beta_{r(k), r^{\prime}}$ represents the large scale fading while each entry of $\mathbf{g}_{r(k), r^{\prime}}[m] \in \mathcal{C}^{N_{T} \times 1}$ denotes the small scale fading represented by an independent and identically distributed (i.i.d.) zero mean complex Gaussian random variable with unit variance. Note that the large scale fading factor $\beta_{r(k), r^{\prime}}^{2}=d_{r(k), r^{\prime}}^{-a}$, where $d_{r(k), r^{\prime}}$ is a distance between UE $r(k)$ and $\mathrm{RU} r^{\prime}$, and $a$ is an attenuation factor.

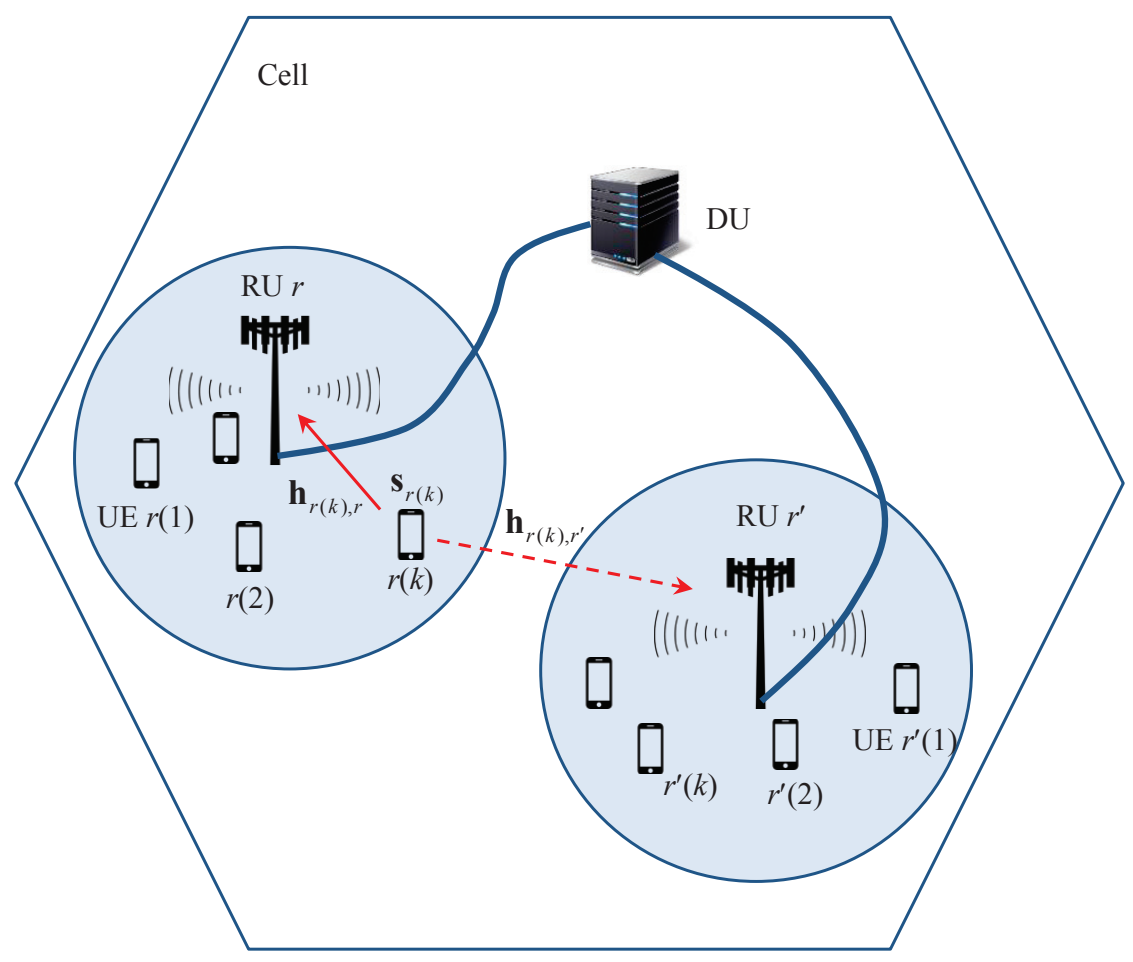

Figure 1. Multi-user m-MIMO configuration in DAS environments.

\section{Conventional Channel Sounding Reference Signal in LTE/LTE-A Systems}

In the following, we briefly introduce the generation of SRS in conventional LTE/LTE-A systems [24,25]. The structure of SRS symbol is illustrated in Figure 2. Basically, the SRS sequence is generated by a cyclic shift of a base sequence, which is obtained from Zadoff-Chu sequence [24,25] as presented below. Let $N_{s c}^{R B}$ be the number of subcarriers per RB, where $N_{s c}^{R B}=12$ in LTE/LTE-A systems, and $L$ be the number of assigned subcarriers for SRS or sounding bandwidth. Let $D$ be the decimation factor which is the number of SRS sequences sharing the allocated sounding bandwidth, where the length of SRS sequence is $M=L / D$. Note that $L$ is the multiple of $N_{s c}^{R B}$, i.e., $L=n \cdot N_{s c}^{R B}$, $1 \leq n \leq N_{R B}^{U L}$, where $N_{R B}^{U L}$ is the uplink system bandwidth in terms of RBs. Let $L_{z}$ denote the length of 
Zadoff-Chu sequence used to generate the base sequence of length $M$, where $L_{z}$ is given by the largest prime number such that $L_{z}<M$.

Let $x_{q}[m]$ denote the $q$-th root Zadoff-Chu sequence defined by

$$
x_{q}[m]=\exp \left\{-j \frac{\pi q m(m+1)}{L_{z}}\right\}, \quad 0 \leq m<L_{z},
$$

where

$$
\begin{aligned}
& q=\lfloor\bar{q}+0.5\rfloor+v(-1)^{\lfloor 2 \bar{q}\rfloor}, \\
& \bar{q}=L_{z}(u+1) / 31
\end{aligned}
$$

with $u \in\{0,1, \cdots, 29\}$ and $v=0$ if $M=n N_{s c}^{R B}, n \leq 5$ and $v=0,1$ if $M=n N_{s c}^{R B}, n \geq 6$. The base sequence $\bar{x}[m]$ is obtained by

$$
\bar{x}[m]=x_{q}\left[m \bmod L_{z}\right], \quad 0 \leq m<M .
$$

Note that base sequences are divided into groups, where $u$ is the group number associated with the physical cell ID and the length of the SRS sequence, and $v$ is the base sequence number within the group. The SRS sequence $x^{(\alpha)}[m]$ of length $M$ is defined by applying a cyclic shift $\alpha, \alpha \in\{0,1 \cdots, 7\}$, to the base sequence $\bar{x}[m]$ as

$$
x^{(\alpha)}[m]=e^{j 2 \pi \frac{\alpha}{L_{c}} m} \bar{x}[m], \quad 0 \leq m<M,
$$

where $L_{c}>7$ to obtain distinct values of $e^{j 2 \pi \frac{\alpha}{L_{c}}}$ for different $\alpha \in\{0,1 \cdots, 7\}$. For any $\alpha_{i}$ and $\alpha_{j}$ chosen from $\{0, \cdots, 7\}$, two sequences $\mathbf{x}^{\left(\alpha_{i}\right)}=\left[x^{\left(\alpha_{i}\right)}[0] \cdots x^{\left(\alpha_{i}\right)}[M-1]\right]^{T}$ and $\mathbf{x}^{\left(\alpha_{j}\right)}=\left[x^{\left(\alpha_{j}\right)}[0] \cdots x^{\left(\alpha_{j}\right)}[M-1]\right]^{T}$ are orthogonal if $\frac{1}{M} \mathbf{x}^{\left(\alpha_{i}\right)}{ }^{H} \mathbf{x}^{\left(\alpha_{j}\right)}=\delta_{i-j}$, where $\delta_{i-j}=1$ if $i=j$ and 0 otherwise, and the superscript $H$ denotes a conjugate transpose of a vector. This condition reduces to $\frac{1}{M} \sum_{m=0}^{M-1} e^{j \frac{2 \pi}{L_{c}}\left(\alpha_{j}-\alpha_{i}\right) m}=\delta_{i-j}$ by using Equation (5) and the property $|\bar{x}[m]|^{2}=1$ for all $m$, which is clear from Equations (2)-(4).

This condition is satisfied only if $\left(\alpha_{j}-\alpha_{i}\right) \frac{M}{L_{c}}$ is an even integer, or, in other words, $L_{c}$ is a factor of $\left(\alpha_{j}-\alpha_{i}\right) \frac{M}{2}$, for $\alpha_{i} \neq \alpha_{j} \in\{0, \cdots, 7\}$. Consequently, $L_{c}$ must be an integer that is greater than 7 and is a factor of $\frac{1}{2} M$.

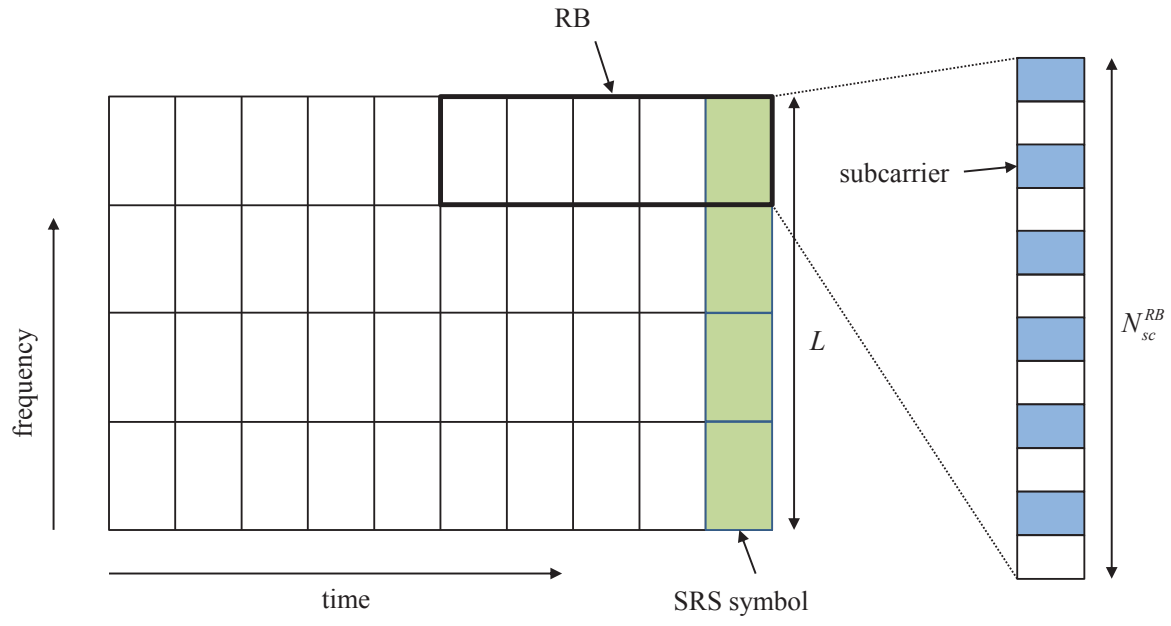

Figure 2. SRS symbol structure.

Multiple SRS sequences are defined from a single base sequence by using different values of $\alpha$ and the decimation factor. In LTE/LTE-A, the decimation factor of two is used and the signal occupies every second subcarrier within the allocated sounding bandwidth. By using distinct SRS 
sequences obtained with different values of $\alpha$ and by using distinct sets of subcarriers as a result of decimation, multiple UEs can estimate their channel gains and can be served by RU simultaneously. In conventional LTE/LTE-A systems using $\alpha \in\{0, \cdots, 7\}$ and the decimation factor of two, we can obtain only 16 orthogonal sequences. In the multi-user systems with higher number of UEs than 16 , we need to design a larger set of SRS sequences having low cross-correlation.

\section{Proposed Channel Sounding Reference Signal for Multi-User Systems}

The lack of orthogonal SRS sequences may cause the pilot contamination problem. The easy way to alleviate this is using longer SRS sequences or wider sounding bandwidth. However, this approach may result in the degraded performance of channel estimation in the frequency selective environment and the lower spectral efficiency and throughput because SRS sequences replace data sequences. Moreover, if the sounding bandwidth is too wide, SRS sequences may occupy the restricted band, which must be prohibited. Thus, we aim to generate a new set of SRS sequences showing reduced pilot contamination without increasing the sounding bandwidth or sequence length. We apply phase rotation to the LTE/LTE-A SRS sequences, which is introduced in Section 3 to generate a new SRS sequence as

$$
x^{(\alpha, s, p)}[m] \triangleq e^{j 2 \pi \frac{s}{M} m} e^{j 2 \pi \frac{p}{L_{p}} m} x^{(\alpha)}[m]=e^{j 2 \pi\left(\frac{s}{M}+\frac{p}{L_{p}}+\frac{\alpha}{L_{c}}\right) m} \bar{x}[m], \quad 0 \leq m<M,
$$

where $0 \leq s<\frac{M}{L_{c}}$ and $0 \leq p<L_{p}$, and the last equality comes from Equation (5). Note that $L_{c}$ is an integer greater than 7 which can divide $\frac{1}{2} M$ as introduced in Section 3 and $L_{p}$ is a prime number which is smaller than $L_{c}$. For a given SRS sequence length $M$, we may generate up to $M$ orthogonal sequences. However, by using Equation (5), we can generate only eight orthogonal sequences with varying $\alpha=0, \cdots, 7$. Thus, we use the phase rotation $e^{j 2 \pi \frac{s}{M} m}, 0 \leq s<\frac{M}{L_{c}}$, together with $e^{j 2 \pi \frac{\alpha}{L_{c}} m}, 0 \leq \alpha<L_{c}$, to generate $M$ orthogonal SRS sequences without incurring pilot contamination. If the number of UEs exceeds $M$, we need to generate extra SRS sequences instead of reusing already generated ones. For this purpose, we apply additional phase rotation $e^{j 2 \pi \frac{p}{L_{p}} m}, 0 \leq p<L_{p}$, where $L_{p}$ needs to be coprime with $M$ and thus be also coprime with $L_{c}$ in order to make the resultant sequences distinct from already generated $M$ sequences. We empirically found that a prime number $L_{p}$ smaller than $L_{c}$ results in a good performance.

By Equations (2)-(4), we can rewrite Equation (6) as

$$
x^{(\alpha, s, p)}[m]= \begin{cases}\exp \left\{j 2 \pi\left(\left(\frac{s}{M}+\frac{p}{L_{p}}+\frac{\alpha}{L_{c}}\right) m-\frac{q m(m+1)}{2 L_{z}}\right)\right\}, & \text { for } 0 \leq m<L_{z}, \\ \exp \left\{j 2 \pi\left(\left(\frac{s}{M}+\frac{p}{L_{p}}+\frac{\alpha}{L_{c}}\right) m-\frac{q\left(m-L_{z}\right)\left(m-L_{z}+1\right)}{2 L_{z}}\right)\right\}, & \text { for } L_{z} \leq m<M .\end{cases}
$$

The correlation of SRS sequences $\mathbf{s}_{r(k)}$ and $\mathbf{s}_{r^{\prime}(j)}$ is defined and expanded as

$$
C_{r(k), r^{\prime}(j)} \triangleq \frac{1}{M} \mathbf{s}_{r(k)}{ }^{H} \mathbf{s}_{r^{\prime}(j)}=\frac{1}{M} \sum_{m=0}^{M-1} s_{r(k)}^{*}[m] s_{r^{\prime}(j)}[m]=\frac{1}{M} \sum_{m=0}^{M-1} e^{-j 2 \pi\left(\frac{s-s^{\prime}}{M}+\frac{p-p^{\prime}}{L p}+\frac{\alpha-\alpha^{\prime}}{L c}\right) m},
$$

where $s_{r(k)}[m]=x^{(\alpha, s, p)}[m], s_{r^{\prime}(j)}[m]=x^{\left(\alpha^{\prime}, s^{\prime}, p^{\prime}\right)}[m]$ and the superscript $*$ represents the complex conjugate of a complex variable. The detailed derivation of Equation (8) is provided in Appendix A. It is clear that $C_{r(k), r^{\prime}(j)}$ obtained with $s=s^{\prime}, p=p^{\prime}$ and $\alpha=\alpha^{\prime}$ corresponds to the auto-correlation of $\mathbf{s}_{r(k)}$ because $\mathbf{s}_{r^{\prime}(j)}=\mathbf{s}_{r(k)}$, where $C_{r(k), r(k)}=\frac{1}{M} \sum_{m=0}^{M-1} e^{j \cdot 0}=1$. The cross-correlation $C_{r(k), r^{\prime}(j)}=0$ if $p=p^{\prime}$ and either $s \neq s^{\prime}$ or $\alpha \neq \alpha^{\prime}$, while $C_{r(k), r^{\prime}(j)} \neq 0$ if $p \neq p^{\prime}$ by the following reason.

Consider $f(\phi)=\frac{1}{M} \sum_{m=0}^{M-1} e^{j 2 \pi \phi m}$, where $f(\phi)=0$ if $\phi M$ is a nonzero integer, and $f(\phi) \neq 0$, otherwise. If $p=p^{\prime}$, the rightmost side of Equation (8) becomes $f\left(\frac{s^{\prime}-s}{M}+\frac{\alpha^{\prime}-\alpha}{L_{c}}\right)$. If $s \neq s^{\prime}$ or $\alpha \neq \alpha^{\prime}$, $\left(\frac{s^{\prime}-s}{M}+\frac{\alpha^{\prime}-\alpha}{L_{c}}\right) M$ is a nonzero integer because $M$ is a multiple of $L_{c}$ as introduced in Section 3 , and thus 
$C_{r(k), r^{\prime}(j)}=f\left(\frac{s^{\prime}-s}{M}+\frac{\alpha^{\prime}-\alpha}{L_{c}}\right)=0$. On the other hand, if $p \neq p^{\prime},\left(\frac{s^{\prime}-s}{M}+\frac{p^{\prime}-p}{L_{p}}+\frac{\alpha^{\prime}-\alpha}{L_{c}}\right) M$ cannot be a nonzero integer because $\left(p^{\prime}-p\right) M$ is not divisible by $L_{p}$, and, thus $C_{r(k), r^{\prime}(j)}=f\left(\frac{s^{\prime}-s}{M}+\frac{p^{\prime}-p}{L_{p}}+\frac{\alpha^{\prime}-\alpha}{L_{c}}\right) \neq$ 0 , where $L_{p}$ and $M$ are coprime and $\left|p-p^{\prime}\right|<L_{p}$.

Consequently, for each $p$, we can generate a set of $M$ orthogonal SRS sequences, where $0 \leq s<\frac{M}{L_{c}}$ and $0 \leq \alpha<L_{c}$ enables the generation of $M$ orthogonal sequences. Applying the phase rotation $e^{j 2 \pi \frac{p}{L p} m}, 0 \leq p<L_{p}$, in Equation (7) enables to obtain $L_{p}$ sets of $M$ orthogonal sequences. Any two sequences obtained with different values of $p$ are mutually correlated.

Distinct SRS sequences generated with different $\alpha, s$ and $p$ by Equation (7) are assigned to different UEs. The first set of $M$ orthogonal sequences generated with $p=0$ are assigned to the first $M$ UEs. Then, the next set of $M$ orthogonal sequences generated with $p=1$ are assigned to the next $M$ UEs. This procedure is repeated by increasing $p$ until all UEs are assigned SRS sequences.

\section{Channel Estimation}

\subsection{Least Square Channel Estimation}

Let $\hat{\mathbf{h}}_{r(k), r}[m]$ denote the estimation of the channel gain $\mathbf{h}_{r(k), r}[m]$. We apply the LS estimation algorithm [26] to the received signal of SRS sequence for the channel estimation. In this process, we assume a block fading with length $M$, by which the channel is considered invariant over consecutive $M$ subcarriers. Then, the estimation of channel gain at the $m$-th subcarrier in a fading block, $\hat{\mathbf{h}}_{r(k), r}[m]$, can also be denoted by $\hat{\mathbf{h}}_{r(k), r}$ and obtained as

$$
\hat{\mathbf{h}}_{r(k), r}[m]=\hat{\mathbf{h}}_{r(k), r}=\frac{1}{M} \sum_{i=0}^{M-1} s_{r(k)}^{*}[i] \mathbf{y}_{r}[i]
$$

By using Equation (1), we can rewrite Equation (9) as

$$
\begin{aligned}
& \hat{\mathbf{h}}_{r(k), r}=\frac{1}{M} \sum_{i=0}^{M-1} s_{r(k)}^{*}[i]\left\{\sum_{j=0}^{K-1} \mathbf{h}_{r(j), r}[i] s_{r(j)}[i]+\sum_{r^{\prime}=0, r^{\prime} \neq r}^{R-1} \sum_{j=0}^{K-1} \mathbf{h}_{r^{\prime}(j), r}[i] s_{r^{\prime}(j)}[i]+\mathbf{n}_{r}[i]\right\} \\
& =\frac{1}{M} \sum_{i=0}^{M-1}\left\{\mathbf{h}_{r(k), r}[i]+\sum_{j=0, j \neq k}^{K-1} \mathbf{h}_{r(j), r}[i] s_{r(k)}^{*}[i] s_{r(j)}[i]+\sum_{r^{\prime}=0, r^{\prime} \neq r}^{R-1} \sum_{j=0}^{K-1} \mathbf{h}_{r^{\prime}(j), r}[i] s_{r^{\prime}(k)}^{*}[i] s_{r^{\prime}(j)}[i]+s_{r(k)}^{*}[i] \mathbf{n}_{r}[i]\right\},
\end{aligned}
$$

where $s_{r(k)}^{*}[i] s_{r(k)}[i]=\left|s_{r(k)}[i]\right|^{2}=1$ is used. If the channel is actually block faded with length $M$, the channel gain $\mathbf{h}_{r(k), r}[m]$ is identical for all $m=0, \cdots, M-1$ and thus, we can represent the channel gain by $\mathbf{h}_{r(k), r}$. Then, Equation (10) can be rewritten as

$$
\hat{\mathbf{h}}_{r(k), r}=\mathbf{h}_{r(k), r}+\sum_{j=0, j \neq k}^{K-1} \mathbf{h}_{r(j), r} C_{r(k), r(j)}+\sum_{r^{\prime}=0, r^{\prime} \neq r}^{R-1} \sum_{j=0}^{K-1} \mathbf{h}_{r^{\prime}(j), r} C_{r(k), r^{\prime}(j)}+\frac{1}{M} \sum_{i=0}^{M-1} s_{r(k)}^{*}[i] \mathbf{n}_{r}[i] .
$$

In case that all SRS sequences of UEs in the cell are mutually orthogonal, we have zero cross-correlation between any two SRS sequences so that Equation (11) is simplified as

$$
\hat{\mathbf{h}}_{r(k), r}=\mathbf{h}_{r(k), r}+\frac{1}{M} \sum_{i=0}^{M-1} s_{r(k)}^{*}[i] \mathbf{n}_{r}[i] .
$$

We define the normalized mean squared error (MSE) of the channel estimation between UE $r(k)$ and RU $r$ at subcarrier $m$ as

$$
\sigma_{M S E, r(k)}^{2}[m]=\frac{E\left\|\hat{\mathbf{h}}_{r(k), r}-\mathbf{h}_{r(k), r}[m]\right\|^{2}}{\beta_{r(k), r}^{2} N_{T}} .
$$


Then, we define the average MSE by

$$
\bar{\sigma}_{M S E}^{2}=\frac{1}{K R M} \sum_{k=0}^{K-1} \sum_{r=0}^{R-1} \sum_{m=0}^{M-1} \sigma_{M S E, r(k)}^{2}[m]
$$

and use this as the performance metric of channel estimation. Note that, if the channel is actually block faded with length $M$, the normalized MSE can be denoted by $\sigma_{M S E, r(k)}^{2}$ and Equation (14) can be simplified as $\bar{\sigma}_{M S E}^{2}=\frac{1}{K R} \sum_{k=0}^{K-1} \sum_{r=0}^{R-1} \sigma_{M S E, r(k)}^{2}$. Under the assumption of block fading with length $M$, the average MSE can be obtained from Equations (11), (13) and (14) as

$$
\begin{aligned}
\bar{\sigma}_{M S E}^{2} & =\frac{1}{K R} \sum_{k=0}^{K-1} \sum_{r=0}^{R-1} \frac{E\left\|\hat{\mathbf{h}}_{r(k), r}-\mathbf{h}_{r(k), r}\right\|^{2}}{\beta_{r(k), r}^{2} N_{T}} \\
& =\frac{1}{K R} \sum_{k=0}^{K-1} \sum_{r=0}^{R-1} \frac{1}{\beta_{r(k), r}^{2}}\left\{\sum_{j=0, j \neq k}^{K-1} \beta_{r(j), r}^{2}\left|C_{r(k), r(j)}\right|^{2}+\sum_{r^{\prime}=0, r^{\prime} \neq r}^{R-1} \sum_{j=0}^{K-1} \beta_{r^{\prime}(j), r}^{2}\left|C_{r(k), r^{\prime}(j)}\right|^{2}+\frac{1}{M} \sigma_{n}^{2}\right\},
\end{aligned}
$$

where the detailed derivation is given in Appendix B.

Let us predict analytically the average MSE under the block fading environment. The large scale fading factors are assumed to be $\beta_{r(k), r}=\beta_{1}$ and $\beta_{r^{\prime}(k), r}=\beta_{2}$ for all $k, r$ and $r^{\prime}, r^{\prime} \neq r$, which means that the large scale fading between UE and serving RU is represented by $\beta_{1}$ while the large scale fading between UE and other neighboring RUs are represented by $\beta_{2}$. We suppose $\beta_{2}<\beta_{1}$ because UE is usually served by a nearly located RU. Then, Equation (15) can be rewritten as

$$
\bar{\sigma}_{M S E}^{2}=\frac{1}{K R} \sum_{k=0}^{K-1} \sum_{r=0}^{R-1}\left\{\sum_{j=0, j \neq k}^{K-1}\left|C_{r(k), r(j)}\right|^{2}+\frac{\beta_{2}^{2}}{\beta_{1}^{2}} \sum_{r^{\prime}=0, r^{\prime} \neq r}^{R-1} \sum_{j=0}^{K-1}\left|C_{r(k), r^{\prime}(j)}\right|^{2}+\frac{\sigma_{n}^{2}}{\beta_{1}^{2} M}\right\} .
$$

We consider the reuse of $M$ orthogonal SRS sequences to UEs repeatedly without applying the phase rotation $e^{j 2 \pi \frac{p}{L p} m}$ in Equation (7). We suppose that each RU in the cell serves the equal number of UEs and distinct set of $\frac{M}{R}$ orthogonal sequences are assigned repeatedly to UEs in each RU. Then, we obtain a correlation matrix $\mathrm{C} \in \mathcal{C}^{K R \times K R}$ of SRS sequences whose $(i, j)$-th entry is defined by

$$
\mathbf{C}_{i j}= \begin{cases}1, & \text { if }|i-j|=k M, \quad k=0, \cdots,\lfloor K R / M\rfloor, \\ 0, & \text { else. }\end{cases}
$$

Every other $\frac{M}{R}$ column and row of C correspond to UEs in the same RU. Due to the reuse of $\frac{M}{R}$ orthogonal sequences for each RU, SRS sequences used for different RUs are always mutually orthogonal. It follows that $C_{r(k), r^{\prime}(j)}=0$ for all $k, j$ and $r \neq r^{\prime}$ and Equation (16) becomes

$$
\bar{\sigma}_{M S E}^{2}=\frac{1}{K R} \sum_{k=0}^{K-1} \sum_{r=0}^{R-1} \sum_{j=0, j \neq k}^{K-1}\left|C_{r(k), r(j)}\right|^{2}+\frac{\sigma_{n}^{2}}{\beta_{1}^{2} M}
$$

From Equation (17) and the allocation rule of orthogonal sequences to UEs introduced above, we obtain

$$
\frac{1}{K R} \sum_{k=0}^{K-1} \sum_{r=0}^{R-1} \sum_{j=0, j \neq k}^{K-1}\left|C_{r(k), r(j)}\right|^{2}= \begin{cases}0, & \text { if } K R \leq M, \\ \frac{1}{K R} \sum_{i=1}^{\left\lfloor\frac{K R}{M}\right\rfloor} 2(K R-i M), & \text { if } K R>M .\end{cases}
$$

Consequently, we can rewrite Equation (18) as

$$
\bar{\sigma}_{M S E}^{2}= \begin{cases}\frac{\sigma_{n}^{2}}{\beta_{1}^{2} M} & \text { if } K R \leq M, \\ \left\lfloor\frac{K R}{M}\right\rfloor\left(2-\frac{M}{K R}\left(\left\lfloor\frac{K R}{M}\right\rfloor+1\right)\right)+\frac{\sigma_{n}^{2}}{\beta_{1}^{2} M^{\prime}} & \text { if } K R>M .\end{cases}
$$


Note that $\bar{\sigma}_{M S E}^{2}$ is independent of $\beta_{2}^{2}$ while it depends on $\beta_{1}^{2}$. Recall that $\beta_{2}^{2}$ is determined by the distance between a UE and its neighboring RU. Thus, the performance of channel estimation obtained by reusing orthogonal sequences repeatedly is not affected by how far neighboring RUs are located from UE. It is also clear from Equation (20) that the average MSE decreases as M grows. Since the number of orthogonal sequences is limited for a given sequence length, the number of UEs served simultaneously in a cell is limited. In the multi-user systems, a larger number of UEs must be accommodated, so we may need to use non-orthogonal sequences for channel sounding.

\subsection{Supplemental Correlation-Aided Channel Estimation}

As the number of UEs grows in the cell, it is impossible to assign orthogonal SRS sequences to all UEs even by using the proposed SRS sequences because the number of orthogonal sequences is limited to $M$. Thus, we propose using the supplemental channel estimation scheme to enhance the channel estimation performance even with correlated SRS sequences. Suppose DU knows SRS sequences and their cross-correlations. We rewrite Equation (11) as

$$
\hat{\mathbf{h}}_{r(k), r}=\sum_{r^{\prime}=0}^{R-1} \sum_{j=0}^{K-1} \mathbf{h}_{r^{\prime}(j), r} C_{r(k), r^{\prime}(j)}+\frac{1}{M} \sum_{i=0}^{M-1} s_{r(k)}^{*}[i] \mathbf{n}_{r}[i],
$$

where $0 \leq r<R$ and $0 \leq k<K$. This can be expressed by using matrix forms as

$$
\hat{\mathbf{H}}=\mathbf{H C}+\mathbf{N},
$$

where $\hat{\mathbf{H}} \in \mathcal{C}^{N_{T} \times K R}, \mathbf{H} \in \mathcal{C}^{N_{T} \times K R}$ and $\mathbf{N} \in \mathcal{C}^{N_{T} \times K R}$ are matrices whose columns are $\hat{\mathbf{h}}_{r(k), r}, \mathbf{h}_{r(k), r}$ and $\mathbf{n}_{r(k), r}$, respectively, $0 \leq r<R$ and $0 \leq k<K$. We let $\mathbf{C} \in \mathcal{C}^{K R \times K R}$ denote a correlation matrix whose entries are $C_{r(k), r^{\prime}(j)}$, where $0 \leq r, r^{\prime}<R$ and $0 \leq k, j<K$. We define two classes of UEs. The first one is the set of UEs that are assigned mutually orthogonal SRS sequences. UEs assigned mutually correlated SRS sequences compose the second class. We reorder $\hat{\mathbf{H}}, \mathbf{H}, \mathbf{C}$ and $\mathbf{N}$ and partition them as $\hat{\mathbf{H}}=\left[\begin{array}{ll}\hat{\mathbf{H}}_{u} & \hat{\mathbf{H}}_{c}\end{array}\right], \mathbf{H}=\left[\begin{array}{ll}\mathbf{H}_{u} & \mathbf{H}_{c}\end{array}\right], \mathbf{C}=\left[\begin{array}{ll}\mathbf{I} & \mathbf{0} \\ \mathbf{0} & \mathbf{A}\end{array}\right]$ and $\mathbf{N}=\left[\begin{array}{ll}\mathbf{N}_{u} & \mathbf{N}_{c}\end{array}\right]$, where submatrices with subscripts $u$ and $c$ correspond to the first and the second class of UEs, respectively, and $\mathbf{A}$ is a non-diagonal matrix. Then, Equation (22) can be rewritten in partitioned forms as

$$
\left[\begin{array}{ll}
\hat{\mathbf{H}}_{u} & \hat{\mathbf{H}}_{c}
\end{array}\right]=\left[\begin{array}{ll}
\mathbf{H}_{u} & \mathbf{H}_{c}
\end{array}\right]\left[\begin{array}{ll}
\mathbf{I} & \mathbf{0} \\
\mathbf{0} & \mathbf{A}
\end{array}\right]+\left[\begin{array}{ll}
\mathbf{N}_{u} & \mathbf{N}_{c}
\end{array}\right],
$$

which implies

$$
\begin{gathered}
\hat{\mathbf{H}}_{u}=\mathbf{H}_{u}+\mathbf{N}_{u}, \\
\hat{\mathbf{H}}_{c}=\mathbf{H}_{c} \mathbf{A}+\mathbf{N}_{c} .
\end{gathered}
$$

It is clear from Equation (24) that $\hat{\mathbf{h}}_{r(k), r}=\mathbf{h}_{r(k), r}+\mathbf{n}_{r(k), r}$ for the first class of UEs. So, $\hat{\mathbf{h}}_{r(k), r}$ is considered to estimate sufficiently the actual channel $\mathbf{h}_{r(k), r}$ of $\mathrm{UE} r(k)$ in the first class. On the other hand, as can be seen from Equation (25), for the second class of UEs, $\hat{\mathbf{h}}_{r(k), r}$ includes the linear combination of other UEs' channels as well. Thus, we need a supplemental procedure to obtain the more reliable channel estimations for UEs in the second class. For this purpose, we propose the correlation-aided channel estimation which applies the MMSE algorithm [27] to the estimated channels for the second class of UEs as the supplemental procedure. We obtain the refined channel estimation $\tilde{\hat{\mathbf{H}}}_{c}$ for UEs in the second class by multiplying the MMSE nulling matrix $\mathbf{W}$ to $\hat{\mathbf{H}}_{c}$ as

$$
\tilde{\hat{\mathbf{H}}}_{c}=\hat{\mathbf{H}}_{c} \mathbf{W},
$$


where

$$
\mathbf{W}=\mathbf{A}^{H}\left(\mathbf{A A}^{H}+\mathbf{B}^{-1} \mathbf{I}\right)^{-1}
$$

and $\mathbf{B}$ is a diagonal matrix whose diagonal entries are $\beta_{r(k), r}^{2}$ corresponding to UEs in the second class. Then, after refinement of $\hat{\mathbf{H}}_{c}$ to $\tilde{\mathbf{H}}_{c}$ by Equation (26), the channel estimation $\hat{\mathbf{H}}$ is replaced by $\hat{\mathbf{H}}=\left[\begin{array}{cc}\hat{\mathbf{H}}_{u} & \tilde{\mathbf{H}}_{c}\end{array}\right]$.

In summary, first, we apply the LS estimation to obtain $\hat{\mathbf{h}}_{r(k), r}$ for all UEs. Next, we define the first and the second classes of UEs based on the correlation matrix $\mathbf{C}$. The first class is defined by UEs whose SRS sequences are mutually orthogonal. The remaining UEs form the second class. Then, for the second class of UEs, we apply additionally the MMSE algorithm to the channel estimation obtained by LS scheme as the supplemental procedure. Finally, we use the result of LS estimation for the first class and the result of supplement estimation for the second class as the channel estimation. If the number of UEs is less than or equal to the number of mutually orthogonal SRS sequences, we only need to perform a LS estimation as introduced in Equation (9). Otherwise, we need to apply the proposed correlation-aided supplemental estimation after performing the LS estimation.

\section{Numerical Results}

We evaluate the performances of the proposed SRS and the proposed supplemental channel estimation algorithm in terms of MSE in the multi-user m-MIMO DAS environment. For comparison, the MSE obtained by using conventional LTE/LTE-A SRS scheme is also shown. As the proposed scheme, we consider the following three types:

(a) using repeatedly $M$ orthogonal sequences generated by varying $\alpha$ and $s$ without applying the phase rotation $e^{j 2 \pi \frac{p}{L p} m}$ in Equation (7),

(b) using the phase rotation $e^{j 2 \pi \frac{p}{L p} m}$ together with varying $\alpha$ and $s$ for SRS generation, but not using the supplemental correlation-aided channel estimation,

(c) using the phase rotation $e^{j 2 \pi \frac{p}{L p} m}$ together with varying $\alpha$ and $s$ for SRS generation, and the supplemental correlation-aided channel estimation as well.

We consider the large scale fading factor between UEs and serving RU as $\beta_{1}$ and the large scale fading factor between UEs and other neighboring RUs as $\beta_{2}$, i.e., $\beta_{r(k), r}=\beta_{1}$ and $\beta_{r(k), r^{\prime}}=\beta_{2}$ for all $k$, $r$ and $r^{\prime} \neq r$. We let $\beta_{2} / \beta_{1}=0.7692$, which comes from the assumption that the distance of UE from serving RU is 1.3 times shorter than the distance from other neighboring RUs, where the attenuation factor is assumed to be $a=2$. We let each RU serve the same number of UEs. We choose $L_{c}=8$ in Equations (5) and (7), and $L_{p}=3$ when applying the phase rotation $e^{j 2 \pi \frac{p}{L p} m}$ in types (b) and (c) of the proposed scheme. We consider a block fading channel, International Telecommunication Union Radiocommunication Sector (ITU-R) Ped A and ITU-R Veh A channels [28], where ITU-R Ped A [29] and ITU-R Veh A channels are examples of frequency selective channels. The simulation parameters used for performance evaluation are listed in Table 1.

We depict the average MSE of channel estimation with respect to the total number of UEs in the cell obtained for block fading channel, ITU-R Ped A channel and ITU-R Veh A channel in Figures $3-5$, respectively. In each figure, we plot the average MSE obtained by using conventional SRS in LTE/LTE-A systems and the proposed scheme of three types.

We also plot the average MSE obtained by applying supplemental MMSE estimation to all UEs instead of applying MMSE selectively only to UEs belonging to the second class, where the phase rotation $e^{j 2 \pi \frac{p}{L p} m}$ is applied and the corresponding curves are marked with 'nonselective supplemental MMSE.' In Figure 3, we include the average MSE predicted analytically by Equation (20) for the proposed scheme of type (a) over block fading channel. 
Table 1. Parameters used in performance evaluation.

\begin{tabular}{cc}
\hline Parameters & Values \\
\hline number of subcarriers $\left(N_{c}\right)$ & 1024 \\
\hline number of subcarriers per RB $\left(N_{s c}^{R B}\right)$ & 12 \\
\hline SRS length $(M)$ & 48 (proposed scheme) \\
\cline { 2 - 2 } & 24 (LTE/LTE-A with a decimation factor of 2) \\
\hline sampling time & $0.1 \mu s$ \\
\hline average SNR $\left(\beta_{1}^{2} / \sigma_{n}^{2}\right)$ & $0 \mathrm{~dB}$ \\
\hline channel model & block fading, ITU-R Ped A, ITU-R Veh A \\
\hline number of RUs $(R)$ & 4 \\
\hline number of RU antennas $\left(N_{T}\right)$ & 8 \\
\hline
\end{tabular}

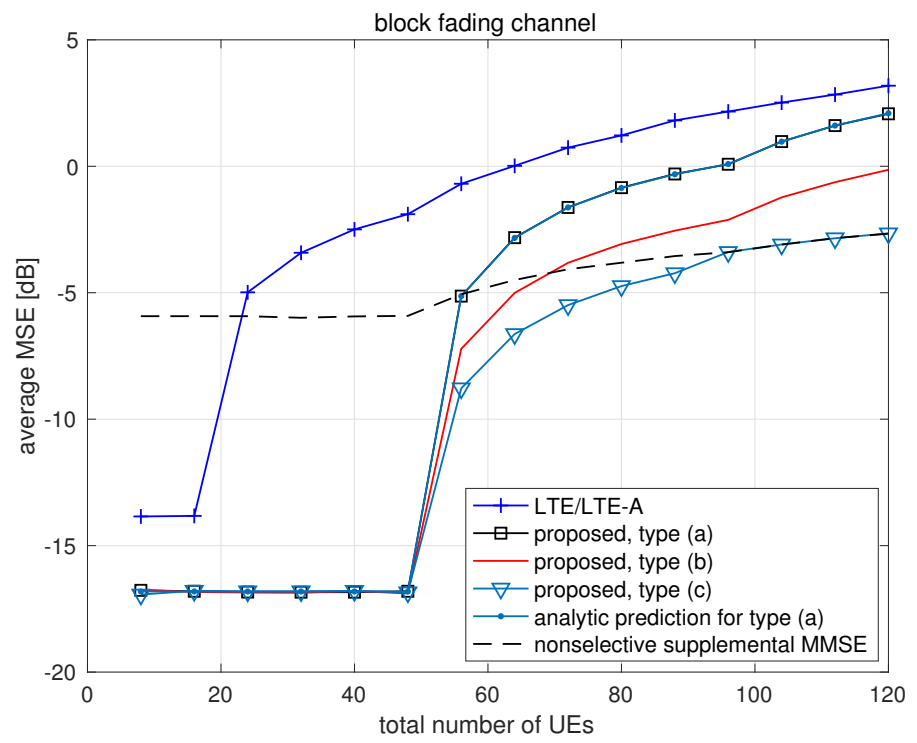

Figure 3. Average MSE of channel estimation with respect to the total number of UEs over block fading channel.

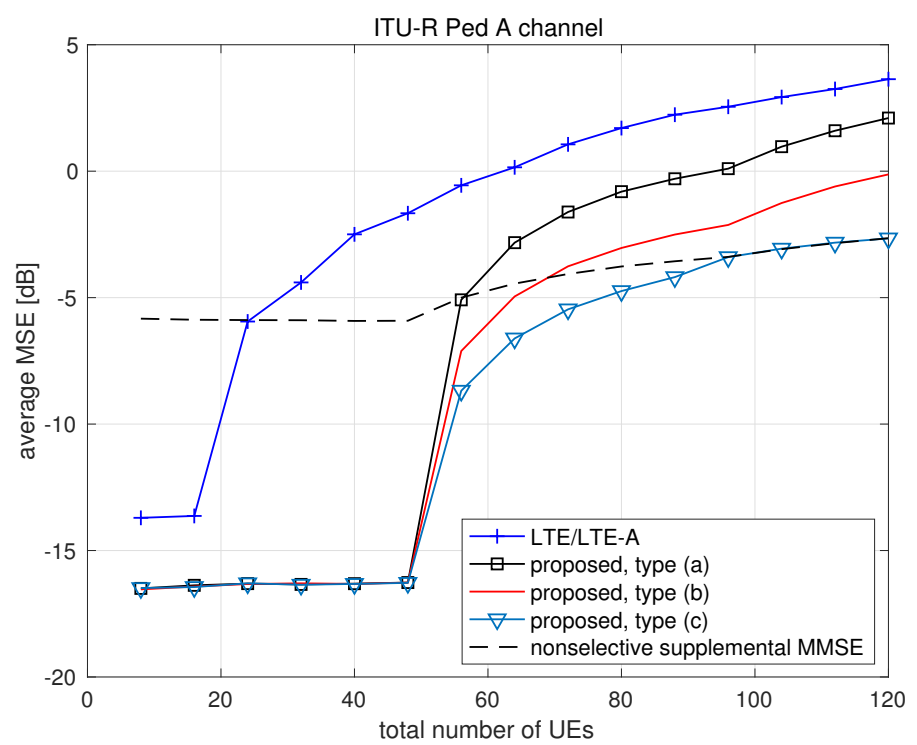

Figure 4. Average MSE of channel estimation with respect to the total number of UEs over ITU-R Ped A channel. 


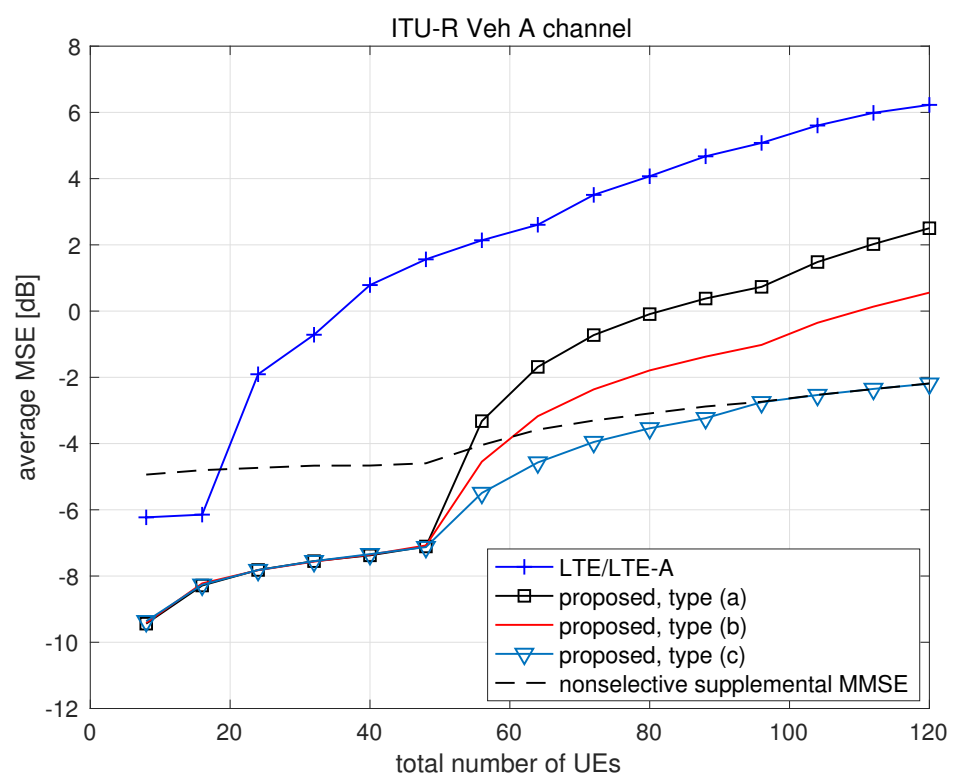

Figure 5. Average MSE of channel estimation with respect to the total number of UEs over ITU-R Veh A channel.

It is observed that the channel estimation MSE obtained by using any SRS scheme rapidly increases when the number of UEs in service exceeds the number of orthogonal SRS sequences, where the conventional LTE/LTE-A scheme and the proposed scheme generate 16 and $M(=48)$ orthogonal SRS sequences, respectively. For more than 48 UEs, the proposed scheme also provides much lower channel estimation MSE than LTE/LTE-A scheme. Even reusing repeatedly $M(=48)$ orthogonal SRS sequences generated in type (a) can lower significantly the channel estimation MSE for any number of UEs. It is observed that applying phase rotation $e^{j 2 \pi \frac{p}{L p} m}$ to SRS generation further lowers the channel estimation MSE, and the use of supplemental selective MMSE estimation can even further improve the channel estimation performance at the cost of increasing complexity. Note that the computational complexity of supplemental selective MMSE estimation is, in general, $O\left(n^{3}\right)$ because it requires the matrix inversion, where $n$ is the dimension of a square matrix A. Considering, as a reference, the case that $M$ orthogonal sequences are repeatedly used to generate SRS sequences, the dimension of $\mathbf{A}$ is determined from Equation (17) as $n=\min (K R, 2 \max (0, K R-M))$. Applying supplemental MMSE estimation nonselectively to all UEs degrades the performance when the number of UEs is not high enough. In Figure 3, the analytic prediction of MSE for block fading channel is observed to match the numerical result very well. It is clear that the proposed scheme of type (c) shows the best channel estimation performance in terms of MSE for all numbers of UEs. The MSE gain of the proposed scheme of type (c) over LTE/LTE-A system is about $6 \mathrm{~dB}$ in block fading and ITU-R Ped A channels, and about $8 \mathrm{~dB}$ in ITU-R Veh A channel when serving 120 UEs through four RUs. The performance gain achieved by using the proposed scheme can be observed in block fading channel as well as frequency selective channel.

Conventional LTE/LTE-A systems may not effectively employ m-MIMO transmission for the service of a large number of UEs mainly due to the pilot contamination problem. However, the proposed SRS and the supplemental channel estimation can relieve the pilot contamination problem in a significant level. It is expected that the proposed scheme can be effectively adopted in multi-user m-MIMO systems.

\section{Conclusions}

In this paper, we proposed a generation of SRS sequences resulting in improved channel estimation performance without increasing the sequence length. The proposed SRS can easily be generated 
by imposing a phase rotation to the base sequence. Even though the proposed SRS sequences are mutually correlated when the number of UEs is higher than the sequence length, it lowers significantly the MSE of channel estimation and thus alleviates the pilot contamination problem. We also proposed a supplemental correlation-aided channel estimation scheme to further improve the channel estimation performance of multi-user m-MIMO technology in DAS environments. It is expected that the proposed SRS scheme and the supplemental channel estimation scheme can be effectively adopted in m-MIMO systems.

Author Contributions: Writing, J.W.L.; Software, S.Y.; Conceptualization, J.W.L.; Supervision, J.W.L.

Funding: This research was supported by the Chung-Ang University Graduate Research Scholarship in 2017 and by the Basic Science Research Program through the National Research Foundation of Korea funded by the Ministry of Education (NRF-2016R1D1A1B03933174).

Conflicts of Interest: The authors declare no conflict of interest.

\section{Appendix A. Derivation of Equation (8)}

With $s_{r(k)}[m]=x^{(\alpha, s, p)}[m]$ and $s_{r^{\prime}(j)}[m]=x^{\left(\alpha^{\prime}, s^{\prime}, p^{\prime}\right)}[m]$, we obtain Equation (8) by using Equation (7) as

$$
\begin{aligned}
C_{r(k), r^{\prime}(j)} \triangleq & \frac{1}{M} \mathbf{s}_{r(k)}{ }^{H} \mathbf{s}_{r^{\prime}(j)}=\frac{1}{M} \sum_{m=0}^{M-1} s_{r(k)}^{*}[m] s_{r^{\prime}(j)}[m] \\
= & \frac{1}{M} \sum_{m=0}^{L_{z}-1} e^{-j 2 \pi\left(\left(\frac{s}{M}+\frac{p}{L_{p}}+\frac{\alpha}{L_{c}}\right) m-\frac{q m(m+1)}{2 L_{z}}\right)} e^{j 2 \pi\left(\left(\frac{s^{\prime}}{M}+\frac{p^{\prime}}{L_{p}}+\frac{\alpha^{\prime}}{L_{c}}\right) m-\frac{q m(m+1)}{2 L_{z}}\right)} \\
& +\frac{1}{M} \sum_{m=L_{z}}^{M-1} e^{-j 2 \pi\left(\left(\frac{s}{M}+\frac{p}{L_{p}}+\frac{\alpha}{L_{c}}\right) m-\frac{q\left(m-L_{z}\right)\left(m-L_{z}+1\right)}{2 L_{z}}\right)} e^{j 2 \pi\left(\left(\frac{s^{\prime}}{M}+\frac{p^{\prime}}{L_{p}}+\frac{\alpha^{\prime}}{L_{c}}\right) m-\frac{q\left(m-L_{z}\right)\left(m-L_{z}+1\right)}{2 L_{z}}\right)} \\
= & \frac{1}{M} \sum_{m=0}^{M-1} e^{-j 2 \pi\left(\frac{s-s^{\prime}}{M}+\frac{p-p^{\prime}}{L_{p}}+\frac{\alpha-\alpha^{\prime}}{L_{c}}\right) m} .
\end{aligned}
$$

\section{Appendix B. Derivation of Equation (15)}

By using Equations (11), (13) and (14), we obtain Equation (15) as

$$
\begin{aligned}
& \bar{\sigma}_{M S E}^{2}=\frac{1}{K R} \sum_{k=0}^{K-1} \sum_{r=0}^{R-1} \frac{E\left\|\hat{\mathbf{h}}_{r(k), r}-\mathbf{h}_{r(k), r}\right\|^{2}}{\beta_{r(k), r}^{2} N_{T}} \\
& =\frac{1}{K R N_{T}} \sum_{k=0}^{K-1} \sum_{r=0}^{R-1} \frac{1}{\beta_{r(k), r}^{2}} E\left\|\sum_{j=0, j \neq k}^{K-1} \mathbf{h}_{r(j), r} C_{r(k), r(j)}+\sum_{r^{\prime}=0, r^{\prime} \neq r}^{R-1} \sum_{j=0}^{K-1} \mathbf{h}_{r^{\prime}(j), r} C_{r(k), r^{\prime}(j)}+\frac{1}{M} \sum_{i=0}^{M-1} s_{r(k)}^{*}[i] \mathbf{n}_{r}[i]\right\|^{2} \\
& =\frac{1}{K R N_{T}} \sum_{k=0}^{K-1} \sum_{r=0}^{R-1} \frac{1}{\beta_{r(k), r}^{2}}\left\{\sum_{j=0, j \neq k}^{K-1} E\left\|\mathbf{h}_{r(j), r}\right\|^{2}\left|C_{r(k), r(j)}\right|^{2}+\sum_{r^{\prime}=0, r^{\prime} \neq r}^{R-1} \sum_{j=0}^{K-1} E\left\|\mathbf{h}_{r^{\prime}(j), r}\right\|^{2}\left|C_{r(k), r^{\prime}(j)}\right|^{2}\right. \\
& =\frac{1}{K R N_{T}} \sum_{k=0}^{K-1} \sum_{r=0}^{R-1} \frac{1}{\beta_{r(k), r}^{2}}\left\{\sum_{j=0, j \neq k}^{M-1} \sum_{i=0}^{K-1} E\left\|\mathbf{n}_{r}[i]\right\|^{2}\right\} \\
& =\frac{1}{K R} \sum_{k=0}^{K-1} \sum_{r=0}^{R-1} \frac{1}{\beta_{r(j), r}^{2}}\left\{\sum_{j=0, j \neq k}^{K-1} \beta_{r(j), r}^{2}\left|C_{r(k), r(j)}\right|^{2}+\sum_{r^{\prime}=0, r^{\prime} \neq r}^{R-1} \sum_{j=0}^{K-1} \beta_{r^{\prime}(j), r}^{2}\left|C_{r(k), r^{\prime}(j)}\right|^{2}+\frac{1}{M} \sigma_{n}^{2}\right\} .
\end{aligned}
$$




\section{References}

1. Ge, X.; Tu, S.; Mao, G.; Wang, C.-X.; Han, T. 5G ultra-dense cellular networks. IEEE Wirel. Commun. 2016, 23, 72-79. [CrossRef]

2. Ge, X.; Cheng, H.; Guizani, M.; Han, T. 5G wireless backhaul networks: Challenges and research advances. IEEE Netw. 2014, 28, 6-11. [CrossRef]

3. Nakamura, T.; Nagata, S.; Benjebbour, A.; Kishiyama, Y.; Hai, T.; Xiaodong, S.; Ning, Y.; Nan, L. Trends in small cell enhancements in LTE Advanced. IEEE Commun. Mag. 2013, 51, 98-105. [CrossRef]

4. Chin, W.H.; Fan, Z.; Haines, R. Emerging technologies and research challenges for 5G wireless networks. IEEE Commun. Mag. 2014, 21, 106-112. [CrossRef]

5. Angelis, C.T.; Chronopoulos, S.K. System performance of an LTE MIMO downlink in various fading environments. In Proceedings of the Ambient Media and Systems, Berlin, Germany, 27-28 January 2011; pp. 36-43._5. [CrossRef]

6. Votis, C.; Christofilakis, V.; Raptis, V.; Tatsis, G.; Chronopoulos, S.K.; Kostarakis, P. Design and analysis of a multiple-output transmitter based on DDS architecture for modern wireless communications. AIP Conf. Proc. 2010, 1203, 421-426. [CrossRef]

7. Marzetta, T. Noncooperative cellular wireless with unlimited numbers of base station antennas. IEEE Trans. Wirel. Comm. 2010, 9, 3590-3600. [CrossRef]

8. Rusek, F.; Persson, D.; Lau, B.; Larsson, E.; Marzetta, T.; Edfors, O.; Tufvesson, F. Scaling up MIMO: Opportunities and challenges with very large arrays. IEEE Signal Process. Mag. 2013, 30, 40-60. [CrossRef]

9. Larsson, E.G.; Tufvesson, F.; Edfors, O.; Marzetta, T.L. Massive MIMO for next generation wireless systems. IEEE Commun. Mag. 2014, 52, 186-195. [CrossRef]

10. Pitarokoilis, A.; Mohammed, S.K.; Larsson, E.G. On the optimality of single-carrier transmission in large-scale antenna systems. IEEE Wirel. Commun. Lett. 2012. 1, 276-279. [CrossRef]

11. Lu, L.; Li, G.Y.; Swindlehurst, A.L.; Ashikhmin, A.; Zhang, R. An overview of massive MIMO: Benefits and challenges. IEEE J. Sel. Top. Signal Process. 2013, 8, 742-758. [CrossRef]

12. Bjornson, E.; Larsson, E.G.; Marzetta, T.L. Massive MIMO: Ten myths and one critical question. IEEE Commun. Mag. 2016, 54, 114-123. [CrossRef]

13. Zuo, J.; Zhang, J.; Yuen, C.; Jiang, W.; Luo, W. Multicell multiuser massive MIMO transmission with downlink training and pilot contamination precoding. IEEE Trans. Veh. Technol. 2016, 65, 6301-6314. [CrossRef]

14. Ge, X.; Zi, R.; Wang, H.; Zhang, J.; Jo, M. Multi-user massive MIMO communication systems based on irregular antenna arrays. IEEE Trans. Wirel. Commun. 2016, 15, 5287-5301. [CrossRef]

15. Gopalakrishnan, B.; Jindal, N. An analysis of pilot contamination on multi-user MIMO cellular systems with many antennas. In Proceedings of the Signal Processing Advances in Wireless Communications (SPAWC), San Francisco, CA, USA, 26-29 June 2011, doi:10.1109/SPAWC.2011.5990435.

16. Heath, R.; Peters, S.; Wang, Y.; Zhang, J. A current perspective on distributed antenna systems for the downlink of cellular systems. IEEE Commun. Mag. 2013, 51, 161-167. [CrossRef]

17. Chen, X.; Zhang, Z.; Chen, H.-H. On distributed antenna systems with limited feedback precoding: opportunities and challenges. IEEE Wirel. Commun. 2010, 17, 80-88. [CrossRef]

18. Yang, C.; Han, S.; Hou, X.; Molisch, A. How do we design CoMP to achieve its promised potential? IEEE Wirel. Commun. 2013, 20, 67-74. [CrossRef]

19. Kobayashi, M.; Jindal, N.; Caire, G. Training and feedback optimization for multiuser MIMO downlink. IEEE Trans. Commun. 2011, 59, 2228-2240. [CrossRef]

20. Elijah, O.; Leow, C.Y.; Abdul Rahman, T.; Nunoo, S.; Zakwoi Iliya, S. A comprehensive survey of pilot contamination in massive MIMO-5G system. IEEE Commun. Surv. Tuts. 2016, 18, 905-923. [CrossRef]

21. Yin, H.; Gesbert, D.; Filippou, M.; Liu, Y. A coordinated approach to channel estimation in large-scale multiple-antenna systems. IEEE J. Sel. Areas Commun. 2013, 31, 264-273. [CrossRef]

22. Ngo, H.Q.; Larsson, E.G. EVD-based channel estimations for multicell multiuser MIMO with very large antenna arrays. In Proceedings of the 2012 IEEE International Conference on Acoustics, Speech and Signal Processing (ICASSP), Kyoto, Japan, 25-30 March 2012; doi:10.1109/ICASSP.2012.6288608.

23. Jose, J.; Ashikhmin, A.; Marzetta, T.L.; Vishwanath, S. Pilot contamination and precoding in multi-cell TDD systems. IEEE Trans. Wirel. Commun. 2011, 10, 2640-2651. [CrossRef] 
24. 3GPP Technical Specification 36.211. Evolved Universal Terrestrial Radio Access (E-UTRA) Physical Channels and Modulation. Available online: www.3gpp.org (accessed on 20 November 2018).

25. 3GPP Technical Specification 36.213. Evolved Universal Terrestrial Radio Access (E-UTRA) Physical Layer Procedures. Available online: www.3gpp.org (accessed on 20 November 2018).

26. Coleri, S.; Ergen, M.; Puri, A.; Bahai, A. Channel estimation techniques based on pilot arrangement in OFDM systems. IEEE Trans. Broadcast. 2002, 48, 223-229. [CrossRef]

27. Jafarkhani, H. Space-Time Coding: Theory and Practice; Cambridge University Press: Cambridge, UK, 2005; pp. 229-230, ISBN 0-521-84291-3.

28. Recommendation ITR-R M.1225. Guidelines for Evaluation of Radio Transmission Technologies for IMT-2000; Int. Telecommun. Union (ITU): Geneva, Switzerland, 1997.

29. Chronopoulos, S.K.; Christofilakis, V.; Tatsis, G.; Kostarakis, P. Performance of turbo coded OFDM under the presence of various noise types. Wirel. Pers. Commun. 2016, 87, 1319-1336. [CrossRef]

(C) 2019 by the authors. Licensee MDPI, Basel, Switzerland. This article is an open access article distributed under the terms and conditions of the Creative Commons Attribution (CC BY) license (http:/ / creativecommons.org/licenses/by/4.0/). 